\title{
DETECTION OF UNCONTROLLED MOTION BEHAVIOR IN HUMAN CROWDS
}

\author{
Vijitha V. A

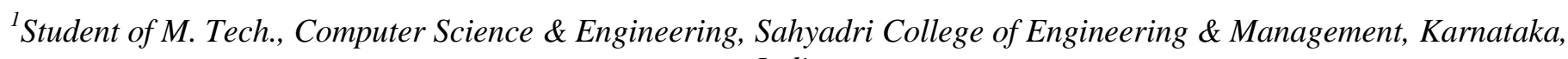 \\ India
}

\begin{abstract}
Analysis of crowd behavior using surveillance videos is an issue for public security. Crowd behavior can be analyzed using two approaches, first one is analyzing individuals behavior in a crowd which is termed as object based and the other one is analyzing the crowd as a whole which is termed as holistic based approach. Identifying and tracking the crowd motion is a major interesting research topic in vision analysis, crowd dynamics and visual surveillance fields. In order to develop the proposed work holistic approach is used. Crowd is the agglomeration of many people in the same area at same time. Music festivals, sports events, pilgrimage are the mass events. In such mass gatherings, the density of the people is more and this may often lead to crowd disasters. Due to this, people will die by suffocating high pressure on their chest. This system is developed to overcome such situations. The proposed system will help the security personnel to evaluate the situation to take necessary actions against the crowd disasters. The decision "if and how to" react solely based on the expert knowledge of the security personnel. The proposed system is based on the optical flow computations and detects the patterns in crowd motion that indicate the dangerous situations. Lucas - Kanade method is used to find the optical flow. Here two input videos are used to conduct the experiment. System will allow only authorized person to use it by entering the "Username and Password". The videos consist of many numbers of frames and this system will extract those frames in the input video. After extracting the frames from the input video, it will divide each frame into sub parts for easy and efficient processing of video sequences and for the respective subpart optical flow vectors are calculated. Threshold optical flow values are chosen in such a way that the tracking of congested region in video is easily performed by comparing it with respective subpart's estimated optical flow values. The system will automatically detect the congested area and also represent the result by plotting the graphs. The proposed system is developed using MATLAB tool. Here videos with avi format are used as the input to the system. This system gives result for the videos taken from the top view and for the crowd with extreme density. This system preserves privacy of people being monitored at the mass event, since it does not detect and track individual people.
\end{abstract}

Keywords: Crowd, Optical Flow, Lucas-Kanade.

\section{INTRODUCTION}

Research in crowd behavior analysis has gained more and more attention in video surveillance domain. Unlike the understanding of actions performed by individuals, the crowd behavior analysis faces more challenges like diverse semantics, complex interaction and various expressions [1]. One of the most important and challenging problems are crowd disasters, which are serious concern during the mass events. Despite huge numbers of security forces, crowd control measures, hundreds of lives are lost in crowd disasters in each year [2].

Mass events are always and have been popular in all over the world. Such mass events are sports events, music festivals and concerts etc. In such mass events numbers of visitors are more and safety measures are more and more important. In such mass gatherings the density of the mass becomes high. Due to the high density, the crowd members lose their individual control and they move involuntarily. People moving involuntarily induce sudden movement of people nearby. These crowd turbulences propagate through the crowd causing people to stumble and fall down [3].
Crowd turbulence is an unanticipated and unintended irregular motion of individuals into different directions due to strong and rapidly changing forces in crowds of extreme density [4]. As a result, most people die by suffocating due to dangerous pressure of up to $4500 \mathrm{~N} / \mathrm{m}$ on their chests. This system helps to prevent such crowd disasters.

Typically, high pedestrian densities occur in locations with limited capacity such as bottlenecks. However, high densities might also be caused by the crowd itself. In a "craze", people rush in order to reach a goal, for instance, an optimal position near the entertainer in a concert. In contrast, crowd stampedes are also often caused by people escaping from a site due to real or perceived danger.

Lack of communication is a major problem in large crowds. While being part of a crowd, individuals can only communicate with their local neighborhood and have a limited view of the whole crowd. This leads to a false perception of movement when people are collapsing and people in the rear perceive a movement and move forward thereby increasing the pressure even more. 
The diversity of human actions is a big issue. The speeds and joint movements for a certain action will differ greatly between individuals. Even the movements of the same person will depend strongly on the circumstance. For these reason, automatic recognition of such actions in real video sequence has been viewed as a challenging task. This system develops a method to recognize specific human actions from video sequences taken in crowded places.

In addition to that, automatic analysis of video streams is a highly challenging research topic. Amongst others, changing illumination conditions, the variability of people's appearances and actions as well as the lack of training data are common problems an automatic surveillance system has to cope with. In the particular case of large human crowds, thousands of people are visible in the scene leading to severe occlusions, thus making conventional tracking approaches are unfeasible.

There are different approaches proposed for the analysis of human behavior. They are divided into three categories namely; people counting, people tracking and behavior understanding. People counting method is to estimate the crowd density, which can be helpful to detect dangerous situations like crowd collapse in an area. People tracking methods, aims at tracking individuals in the crowd and individual trajectories are used to detect the abnormalities in the scene. In case of crowd analysis, tracking is not an appropriate method because of the difficulty in segmenting and lack of accuracy in tracking individual, mostly due to the occlusions [5]. Behavior understanding is further divided into two they are, object-level approaches and holistic approaches. Object level approaches mainly to detect the individuals in the crowd and analyzing their behaviors. In holistic approach, crowd is treated as a single entity. Holistic approach is used for the proposed system [6].

This system studies the pedestrian behavior in human crowds. Here the main contribution is observation and the quantitative study of motion patterns in congested areas as well as the development of a computer vision system that makes use of these observations and is able to detect unusual events and critical situations such as congestions in human crowds. It is the system for the detection and early warning of uncontrolled situations during mass events.

Proposed system analyzes video footage with the help of computer vision techniques. Here it aims at detecting phase changes caused by changing pedestrian flow due to congestions. In order to detect the motion patterns optical flow method is used here. However, in this scenario, motion patterns of a crowd are composed of different actions performed by many different people. Therefore, here decompose complex motion patterns into simpler motion patterns. For the optical flow, Lucas-Kanade filter is used.

The frames in the input video are sub divided into different subparts and for each part the optical flow is applied. In congested areas, the crowd is moving slower or has come to a halt. When observing such a situation from above, one can estimate the people's velocities by considering the magnitude of optical flow vectors. So depending on the magnitude values, will get the optical flow for each subpart and determine whether there is congestion or not in the video.

Table -1: Some of the Crowd Stampedes[7]

\begin{tabular}{|l|l|l|}
\hline Year & Location & $\begin{array}{l}\text { Deaths } \\
\text { Occurred }\end{array}$ \\
\hline 2013 & $\begin{array}{l}\text { Stampede at Ratangarh Mata } \\
\text { Temple in Datia district, } \\
\text { Madhya Pradesh, India. }\end{array}$ & $\begin{array}{l}\text { More than } \\
100\end{array}$ \\
\hline 2011 & $\begin{array}{l}\text { Stampede near Sabarimala } \\
\text { temple in Kerala, India. }\end{array}$ & $\begin{array}{l}\text { More than } \\
100\end{array}$ \\
\hline 2010 & $\begin{array}{l}\text { Love Parade Disaster at an 20 } \\
\text { Duisburg Germany }\end{array}$ & More than \\
\hline 2008 & $\begin{array}{l}\text { Overcrowded children's dance } \\
\text { hall in Tanzania }\end{array}$ & 20 \\
\hline 2008 & $\begin{array}{l}\text { Chamunda Devi stampede at } \\
\text { the Chamunda Devi temple } \\
\text { Jodhpur, India. }\end{array}$ & 147 \\
\hline 2007 & \begin{tabular}{l} 
Train station at northern India \\
\hline
\end{tabular} & More than 14 \\
\hline
\end{tabular}

\section{LITERATURE SURVEY}

There are two approaches for the behavior understanding of the crowd. They are namely object-based and holistic approach. Object-based approaches perform the crowd behavior understanding through the segmentation or by detecting the individuals to analyze the group behaviors. This approach considers a crowd as a collection of individuals. In more denser scenes it is difficult to track the individual components in the crowd therefore the second approach that is holistic approach is well suited for the proposed system [5].This approach try to obtain the global information like main crowd flows and they ignore the local information such as a person moving against the flow.

Barbara Krausz et. al [3], has developed a system that automatically detects the motion behavior of crowd based on the optical flow fields to determine major motion patterns and motion directions in the crowd. They used quadratic polynomials to calculate the optical flow fields. They computed dense optical flow fields using the method proposed by Farneback, that is quadratic polynomials are used to estimate translations of a local neighborhood and motion vectors are determined from polynomial expansion coefficients.

Guler Puren [6] has developed a method for automated analysis of a crowd behavior using surveillance videos and detect the dangerous crowds and where they are headed. For the analysis of crowd they used behavior understanding. Holistic approach is used to develop a real-time abnormality in crowds using scale invariant feature transform (SIFT) based features and unsupervised machine learning techniques. 
The work by Drik Helbing and Peter Molnar [8] is Social force model. It has been shown that pedestrian motion can be described as if they would be subject to social forces. These forces are a measure for the internal motivations of the individuals to perform certain actions. In this model computer simulation of pedestrian groups demonstrated, 1.the development of lanes consisting of pedestrians who walk into the same direction, 2.oscillatory changes of the walking direction at narrow passages. These spatio-temporal patterns arise due to the nonlinear interactions of pedestrians. They are not the effect of strategical considerations of the individual pedestrians since they were assumed to behave in a rather "automatic" way.

The work by C. Bursttedde et. al [9] is Cellular automata model. It is developed to simulate pedestrian behavior. Here, the key mechanism is the introduction of the floor field which acts as a substitute for pedestrian intelligence and leads to collective phenomena. Long-range interactions between the pedestrians are mediated by floor field. It modifies the transition rates to neighboring cells. Floor field can be discrete or continuous, which is subject to diffusion and decay. This can be modified by the motion of the pedestrians. The main objective of this study is to show that the introduction of floor field is sufficient to model collective effects and self -organization encountered in pedestrian dynamics. In this model computational effort increases linearly with the increase of system size.

Weina Ge [10] has developed an automated pedestrian detection and tracking system. This system automatically detects small groups of individuals who are travelling together. System can extract trajectories from video and that hierarchical clustering can detect small groups of people travelling together. Their results demonstrate that automated tracking is capable of providing quantitative characterization of real crowds faster and with similar accuracy as human observation, providing a new methodology for the empirical study of social behavior.

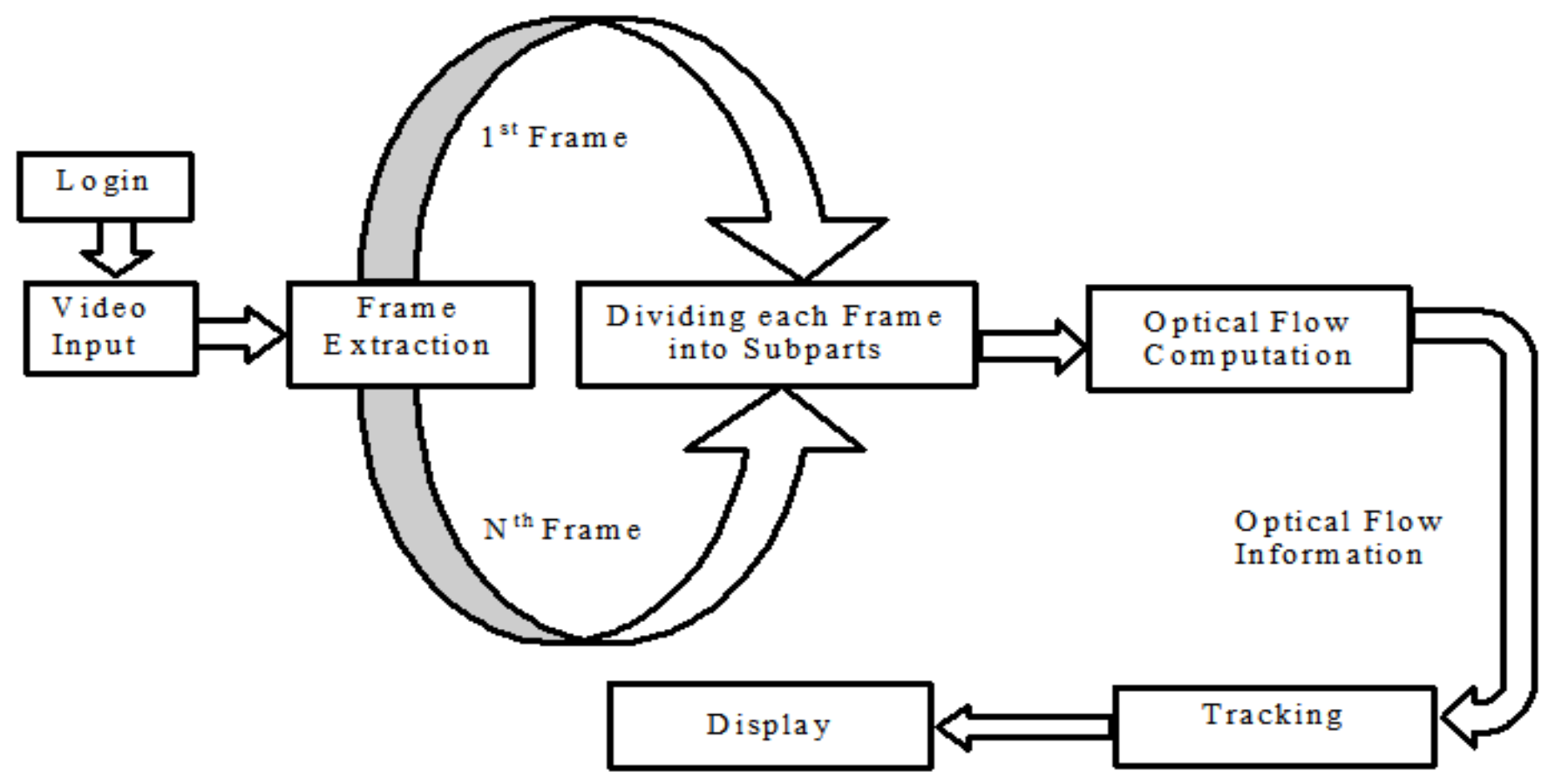

Fig -1: Block Diagram

\section{BLOCK DIAGRAM}

Here video input is given in avi format. Videos contain many number of frames, therefore next step is to extract those frames from the input video that is shown in the Fig- 1 as 1 to $\mathrm{N}$ frames. After extracting each frame from the input video it is divided into sixteen subparts, which is shown in the Fig-2. Frames are divided for easy and efficient processing of video sequences. Next step is to compute the optical flow for each subpart of the frame. For the optical flow computation Lucas-Kanade method is used here. Next step is to track the congested area based on the information obtained from the optical flow computation.

\begin{tabular}{|l|l|l|l|}
\hline 1 & 2 & 3 & 4 \\
\hline 5 & 6 & 7 & 8 \\
\hline 9 & 10 & 11 & 12 \\
\hline 13 & 14 & 15 & 16 \\
\hline
\end{tabular}

Fig -2: Sixteen subparts of the frame 


\subsection{Optical Flow Computation}

Optical flow is referred to a visual phenomenon which is experienced when an object is moving at different speed than the observer. In other words it could be defined as the motion of every pixel in the image between two consecutive video frames. Optical flow method is commonly used to detect moving human being or objects independently based on their individual velocities from camera installed on movable platform [11].

There are two causes that produce the motion of the pixels in the image. First one is the motion of the vision system which is used to represent the image and the second one is the motion of the objects represented in pixels in the image. Therefore optical flow is the combination of those motions except when one of two causes is not present. Optical flow is also depends on the relative distance between the object and the camera.

The Lucas-Kanade approach is popular and robust differential technique for optical flow computation. This technique takes a major assumption that the flow does not vary much in the surrounding region of the pixel which is considered and basic optical flow equations are solved for every pixels in that region by using least square method.

Lucas-Kanade method calculates the motion between two successive frames that are taken at time $\mathrm{t}$ and $t+\partial t$ for every pixel position [12].

In the congested area, the people either move very slowly or stops moving itself. Here the scenario is analyzed for the top-view portion of video. The crowd velocity is estimated by assuming the magnitude of the optical flow vectors. Magnitude value will be zero if the people stops moving or there is no motion otherwise it is nonzero. If the person is walking in a non-congested area then the magnitude value will be more than fifty. A threshold optical flow vectors are chosen for comparing each part already estimated optical flow fields with them. Therefore, if the velocity values are less than the chosen threshold then there is no movement in that particular region and it is a congested area. Else we conclude that people in that area moves freely and there will not be any congestion. Here, it will consider the magnitude value to detect the congested area in the input video.

\section{CONCLUSIONS \& FUTURE WORK}

The system automatically detects the congested area and the system mainly focuses on detection of the motion in the crowd using the optical flow computation method and dividing the frame sequences. Depending on the magnitude values, it tracks the congested area in the crowd. By using this, system security personnel can evaluate the situation to take necessary actions. The decision is "if and how to" react solely based on the expert knowledge of the security personnel. This is a system for decision support could help to prevent accidents or crowd disasters. This system preserves privacy of people being monitored at the mass event, since it does not detect and track individual people.
The limitations of the proposed system is that, the method used here will provide proper results only if the input video is taken from camera which is not moving while capturing the videos and the input video should be taken from the top view.

The system can be enhanced by using various techniques to improve the tracking the congested area in the video. This is the tracking application based on single view (top- view). In future, it can be enhanced by taking the videos from multiple views. This system gives the proper result only if the input video is taken from the camera which is not moving, this limitation can be overcome by using some other techniques.

\section{REFERENCES}

[1]. Yanhao Zhang, Lei Qin, Hongxum Yao, and Qingming Huang, "Abnormal Crowd Behavior Detection Based on Social Attribute-Aware Force Model," International conference on Image Processing, 2012, pp. 2689-2692.

[2]. Dirk Helbeing and Ander Johansson, "The Dynamics of Crowd Disasters: An Empirical Study," in Phys. Rev. E, vol. 75,2007, pp. 046109.

[3]. Barbara Krausz, Christian Bauckhage, Fraunhofer IAIS , "Automatic Detection of Dangerous Motion Behavior in Human Crowds, "in 8th IEEE International Conference on Advanced Video and Signal-Based Surveillance,2011, pp. 224-229.

[4]. D Helbeing and A. Johansson, "Pedestrian, Crowd and Evacuation Dynamics,"in: Meyers, Robert A (ed.) Encyclopedia of Complexity and Systems Science (Springer, Heidelberg) 2009, pp. 6476-6495.

[5]. Julio Cezer Silveira Jacques Junior, Soraira Raupp Musse and Claudio Rosito Jung," Crowd Analysis using Computer Vision Techniques," in IEEE Signal Processing Magazine ,2010, pp. 66-77.

[6]. Puren Guler, "Automated Crowd Behavior Analysis for Video Surveillance Applications," M.S. Thesis, Dept. of Information Systems, Middle East Technical University, 2012.

[7].

Online

available http://en.wikipedia.org/wiki/List_of_human_stampedes\#21s t_century

[8]. Drik Helbing and Peter Molnar, "Social Force Model for Pedestrian Dynamics", in Physical Review E vol. 51 no. 5,May 1995, pp. 4282.

[9]. C. Burstedde, K. Klauck, A. Schadschneider, J. Zittartz, "Simulation of Pedestrian Dynamics using a TwoDimensional Cellular Automaton," in Physica A 295 , 2001, pp. 507-525.

[10]. Weina Ge, Robert T Collins and R. Barry Runback, "Vision-Based Analysis of Small Groups in Pedestrian Crowds," in Pattern Analysis and Machine Intelligence, IEEE Transactions on vol.34, no.5, 2012 , pp. 1003-1016.

[11]. Sarvesh Vishwakarma and Anupam Agrawal., "A Survey on Activity Recognition and Behavior Understanding in Video Surveillance," in The Visual Computer 29, no. 10, 2013, pp. 983-1009.

[12]. Nan Lu, Jihong Wang, Li Yang and Henry Wu,'Motion Detection Based on Accumulative Optical 
Flow and Double Background Filtering," in Proceedings of the World Congress on Engineering, London , U.K, vol I, July 2007.

\section{BIOGRAPHIE}

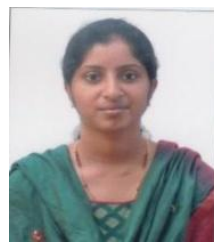

Vijitha V. A , Completed B. E. in Information Science \& Engineering and presently pursuing Mtech in Computer Science \& Engineering from Sahyadri College of Engineering \& Management, Mangalore 